\title{
Metodologia de Controle Inverso Adaptativo Indireto via Algoritmo FASS-NLMS
}

\author{
Rodrigo Possidônio Noronha ${ }^{1}$ \\ Instituto Federal de Educação, Ciência e Tecnologia do Maranhão, Imperatriz, MA
}

Resumo. Uma questão fundamental em aplicações envolvendo filtros adaptativos é que durante a atualização da estimativa do vetor de pesos por um algoritmo de otimização seja obtido um bom trade-off entre a velocidade de convergência e o erro médio quadrático no regime estacionário. Nesse sentido, visando desenvolver um algoritmo de otimização baseado em gradiente descendente estocástico com uma rápida velocidade de convergência e um pequeno erro médio quadrático no regime estacionário, é proposta uma nova versão do Normalized Least Mean Square (NLMS) com o tamanho de passo adaptado por um Sistema de Inferência Fuzzy Mamdani. Para validação do algoritmo proposto foi realizado o projeto de Controle Inverso Adaptativo Indireto (do inglês, IAIC - Indirect Adaptive Inverse Control) para uma planta de fase não mínima na presença de um sinal de distúrbio adicionado ao sinal de controle.

Palavras-chave. Controle Inverso Adaptativo, Filtro Adaptativo, Gradiente Descendente Estocástico, NLMS, Sistemas Fuzzy.

\section{Introdução}

$\mathrm{Na}$ tentativa de solucionar problemas da teoria de sistemas de controle adaptativo do ponto de vista da teoria de filtros adaptativos, foi proposto a técnica de Controle Inverso Adaptativo (do inglês, AIC - Adaptive Inverse Control) [12]. O AIC é realizado através de técnicas de modelagem inversa, no qual a função de transferência do controlador se aproxima, a cada instante de tempo, da inversa da função de transferência da planta. Através da modelagem inversa da planta, é possível, sem feedback, controlar a dinâmica da planta e rejeitar distúrbios. Existem várias contribuições ao AIC publicadas na literatura, como em [10] que é proposta a utilização do método de regressão de máquina de vetores de suporte (do inglês, SVM - Support Vector Machine) baseado em kernel cache, para a obtenção do modelo inverso da planta do AIC. Em [8], foi proposto a utilização de uma rede neuro-fuzzy tipo 2 para o projeto de AIC para aplicação em plantas não lineares.

Para as técnicas de AIC, o vetor de pesos da função de transferência inversa da planta é atualizado em função da minimização de um índice de performance por um algoritmo de otimização baseado, por exemplo, no método do gradiente descendente estocástico. Na literatura, para o projeto de AIC, já foram utilizados os seguintes algoritmos adaptativos baseados no método do gradiente descendente estocástico: Least Mean Square (LMS) [11], Normalized Least Mean Square (NLMS) [7] e Affine Projection (AP) [3]. O algoritmo NLMS ao contrário do LMS, soluciona o problema de sensibilidade à dimensão do vetor de entrada pela normalização com a potência desse sinal. Embora o AP seja derivado do NLMS para utilização em sinais fortemente correlacionados, o AP tem um custo computacional elevado devido utilizar uma matriz de projeções do sinal de

\footnotetext{
${ }^{1}$ rodrigo.noronha@ifma.edu.br
} 
entrada para atualizar o vetor de pesos. Nesse sentido, a proposta realizada neste trabalho se limita à contribuição do algoritmo NLMS ao projeto de IAIC.

O desempenho do algoritmo NLMS é influenciado pelo tamanho de passo definido no intervalo de estabilidade $0<\mu<2$, de tal forma que se o tamanho de passo é grande, a velocidade de convergência do NLMS será rápida, mas o erro médio quadrático (do inglês, MSE - Mean Square Error) no regime estacionário será grande. Se o tamanho de passo é pequeno, a velocidade de convergência do NLMS será lenta, mas o MSE no regime estacionário será pequeno [2]. Nesse sentido, através de uma correta seleção de valores para o tamanho de passo é possível obter um bom trade-off entre a velocidade de convergência e o MSE no regime estacionário [1]. As principais abordagens que tratam de solucionar esse problema utilizam o tamanho de passo variável (do inglês, VSS - Variable Step Size), baseado em algum critério de ajuste para que durante os primeiros instantes de tempo o tamanho de passo seja grande e com a proximidade do fim o tamanho de passo seja pequeno. Algumas propostas para o ajuste do VSS publicadas na literatura podem ser vistas em $[5,6]$. Para realizar o ajuste do VSS essas propostas necessitam de parâmetros adicionais, tais como: fator de amortecimento, parâmetros de ponderação, entre outros. Além disso, geralmente necessitam de medidas estatísticas de alta ordem que nem sempre estão disponíveis para sistemas que operam em tempo real, em que as informações dinâmicas do sistema são disponibilizadas em grandes quantidades na forma de fluxo de dados que necessitam ser rapidamente processados e logo em seguida descartados. Nota-se ainda, que o ajuste do VSS é geralmente dependente da natureza do problema.

Uma alternativa para ajustar o VSS visando um bom trade-off é através de um Sistema de Inferência Fuzzy Mamdani (do inglês, MFIS - Mamdani Fuzzy Inference System). Por meio de um MFIS, é possível modelar o comportamento dinâmico do tamanho de passo de forma independente de parâmetros adicionais, medidas estatísticas de alta ordem do sistema e da natureza do problema. Existem poucas propostas de NLMS com o tamanho de passo ajustado por um MFIS publicadas na literatura. Em [9], foi proposta a utilização de um MFIS para ajustar o tamanho de passo com objetivo de equalizar canais. Em [4] o algoritmo NLMS é aplicado várias vezes utilizando diferentes tamanhos de passo, onde a partir um sistema neuro-fuzzy, é obtido o tamanho de passo ótimo. O problema da metodologia proposta em [4] é o custo computacional para a etapa de treinamento e o tamanho de passo não ser adaptativo. Na metodologia proposta em [9] o tamanho de passo é adaptado somente em função do erro quadrático, não sendo levado em consideração nenhuma informação sobre a quantidade de instantes de tempo necessários para a convergência. A quantidade de instantes de tempo é um importante parâmetro para a otimização, uma vez que se espera que ao fim da simulação ocorra a convergência do vetor de pesos do filtro adaptativo.

Nesse trabalho, é proposta uma nova versão do algoritmo NLMS, intitulada Fuzzy Adaptive Step Size - Normalized Least Mean Square (FASS-NLMS). O tamanho de passo é adaptado em função do erro quadrático e do instante de tempo normalizado pelo método Min-Max, que são as entradas do MFIS. Por meio da combinação das proposições fuzzy do antecedente e consequente através de conectivos lógicos, é possível obter um bom trade-off entre a velocidade de convergência e o MSE no regime estacionário. Para validação da metodologia proposta foi realizado o projeto de IAIC aplicado em uma planta de fase não mínima na presença de um distúrbio. Até o presente momento, de acordo com os estudos bibliográficos realizados pelo autor, a literatura não contém nenhuma abordagem do algoritmo NLMS com o tamanho de passo descrito por um sistema fuzzy utilizado para o projeto de IAIC. Com objetivo de comparação de desempenho, os resultados obtidos com o algoritmo FASS-NLMS foram comparados com a versão tradicional do algoritmo NLMS, em que foi possível obter avanços promissores. Esse artigo é organizado como segue: na Seção 2 são formuladas as equações de diferença do IAIC; na Seção 3 é apresentado o algoritmo FASS-NLMS; na Seção 4 são apresentados os resultados obtidos pela aplicação do IAIC projetado pelo algoritmo FASS-NLMS a uma planta de fase não mínima na presença de um sinal de distúrbio. 


\section{Controle Inverso Adaptativo Indireto}

Seja a relação entre o sinal de controle $u(k)$ e o sinal de saída de uma planta $y(k)$ discreta no tempo e estável ou estabilizável, dada por:

$$
y(k)=q^{-d} \frac{B\left(q^{-1}\right)}{A\left(q^{-1}\right)} u(k)=P\left(q^{-1}\right) u(k),
$$

em que, $P\left(q^{-1}\right)$ é o modelo da planta, onde $\left[a_{1}, a_{2}, \ldots, a_{n}\right] \in \mathbb{R}^{n \times 1}$ e $\left[b_{1}, b_{2}, \ldots, b_{m}\right] \in \mathbb{R}^{m \times 1}$ são os parâmetros do modelo, com $n, m \in \mathbb{N}$. O operador $q^{(-\bullet)}$ é um operador de atraso, tal que, por exemplo, $a_{n} q^{-n} u(k)=a_{n} u(k-n)$. O polinômio $A\left(q^{-1}\right)=1+a_{1} q^{-1}+a_{2} q^{-2}+\ldots+a_{n} q^{-n}$, $B\left(q^{-1}\right)=1+b_{1} q^{-1}+b_{2} q^{-2}+\ldots+b_{m} q^{-m}, k \in \mathbb{N}$ que representa o instante de tempo ou amostra e $d \in \mathbb{N}$ que representa o atraso do sistema. É importante notar que a planta é considerada causal ${ }^{2} \mathrm{e}$ de fase não mínima ${ }^{3}$. A estrutura do IAIC utilizada nesse trabalho foi proposta em [12], conforme a Figura 1.

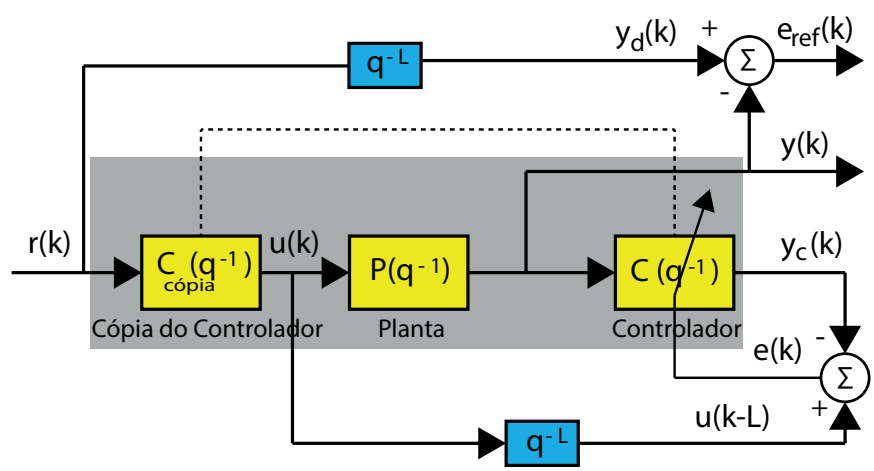

Figura 1: Diagrama de Blocos do IAIC.

Na Fig. 1 o controlador é representado por $C\left(q^{-1}\right)$, que idealmente é igual ao modelo inverso da planta $P\left(q^{-1}\right)$, obtido através de técnicas de modelagem inversa. Com o objetivo de obter uma configuração feedforward com a planta $P\left(q^{-1}\right), C\left(q^{-1}\right)$ é então copiado para à esquerda da planta, obtendo-se $C_{\text {cópia }}\left(q^{-1}\right)$ que é o IAIC, obtido de forma indireta através de $C\left(q^{-1}\right)$. Para filtragem adaptativa linear, a configuração feedforward não altera significativamente a rastreabilidade da dinâmica inversa da planta, uma vez que $C_{\text {cópia }}\left(q^{-1}\right) P\left(q^{-1}\right) \cong P\left(q^{-1}\right) C\left(q^{-1}\right)$. Plantas com atraso puro de tempo não podem responder instantaneamente a uma ação de controle. Para solucionar esse problema, é incorporada à estrutura do IAIC um bloco de atraso $q^{-L}$, tal que $L \cong(M+d+m) / 2$ [7]. Considerando-se que o IAIC seja representado por um filtro Finite Impuse Response (FIR) adaptativo de ordem $M$. Nesse caso, a estimativa do modelo inverso adaptativo $\hat{C}\left(q^{-1}\right)$ à direita da planta e a estimativa do modelo inverso adaptativo $\hat{C}_{\text {cópia }}\left(q^{-1}\right)$ à esquerda da planta, são representados por um filtro FIR adaptativo, em que $\boldsymbol{\Theta}(k)=\left[\theta_{1}, \theta_{2}, \ldots, \theta_{M}\right] \in \mathbb{R}^{M \times 1}$ é o vetor de pesos. Seja então, o sinal $y_{c}(k)$ a saída do filtro FIR adaptativo $\hat{C}\left(q^{-1}\right)$ dado por:

$$
y_{c}(k)=\hat{C}\left(q^{-1}\right) y(k),
$$

uma vez que $\hat{C}\left(q^{-1}\right)=\theta_{0}+\theta_{1} q^{-1}+\theta_{2} q^{-2}+\ldots+\theta_{M-1} q^{-M}$, a saída do filtro FIR adaptativo $\hat{C}\left(q^{-1}\right)$ é reescrita como $y_{c}(k)=\theta_{0} y(k)+\theta_{1} y(k-1)+\ldots+\theta_{M-1} y(k-M)$. De forma vetorial,

\footnotetext{
${ }^{2}$ Os graus dos polinômios $A\left(q^{-1}\right)$ e $B\left(q^{-1}\right)$ são definidos como $n \geq m$.

${ }^{3} \mathrm{O}$ polinômio $B\left(q^{-1}\right)$ contém pelo menos uma raiz não localizada no interior do círculo de raio unitário. Dessa forma, o modelo inverso da planta será instável.
} 
a saída do filtro FIR adaptativo $\hat{C}\left(q^{-1}\right)$ é dada por $y_{c}(k)=\boldsymbol{\Psi}^{T}(k) \boldsymbol{\Theta}(k)=\boldsymbol{\Theta}^{T}(k) \boldsymbol{\Psi}(k)$, em que $\boldsymbol{\Psi}(k)=[y(k), y(k-1), \ldots, y(k-M)] \in \mathbb{R}^{M \times 1}$ é o vetor de regressores ou vetor de sinal de saída da planta.

O erro $e(k)$ é obtido entre a saída do filtro FIR adaptativo $\hat{C}\left(q^{-1}\right)$ e o sinal de controle $u(k)$ da planta $P\left(q^{-1}\right)$, que é utilizado para atualizar a estimativa do vetor de pesos $\boldsymbol{\Theta}(k)$. Para obter $e(k)$ inicialmente é necessário calcular o sinal controle $u(k)$ obtido na saída de $\hat{C}_{c o ́ p i a}\left(q^{-1}\right)$, dado por $u(k)=\hat{C}_{\text {cópia }}\left(q^{-1}\right) r(k)$. Uma vez que $\hat{C}_{\text {cópia }}\left(q^{-1}\right)=\theta_{0}+\theta_{1} q^{-1}+\theta_{2} q^{-2}+\ldots+\theta_{M-1} q^{-M}$, o sinal de controle no instante de tempo $k$ é dado por $u(k)=\theta_{0} r(k)+\theta_{1} r(k-1)+\ldots+\theta_{M-1} r(k-M)$, em que $r(k)$ é o sinal de referência. De forma vetorial o sinal de controle $u(k)$ é dado por:

$$
u(k)=\mathbf{R}^{T}(k) \Theta(k)=\boldsymbol{\Theta}^{T}(k) \mathbf{R}(k),
$$

em que $\mathbf{R}(k)=[r(k), r(k-1), \ldots, r(k-M)] \in \mathbb{R}^{M \times 1}$ é o vetor de regressores ou vetor de sinal de referência. Após obtido o sinal de controle $u(k)$, o erro $e(k)=q^{-L} u(k)-y_{c}(k)$ é reescrito como $e(k)=u(k-L)-\left[\theta_{0} y(k)+\ldots+\theta_{M-1} y(k-M)\right]$. A medida que $\lim _{k \rightarrow \infty}(e(k))^{2} \rightarrow 0$, $\lim _{k \rightarrow \infty} y(k) \rightarrow y_{d}(k)$ e, consequentemente $\lim _{k \rightarrow \infty}\left(e_{r e f}(k)\right)^{2} \rightarrow 0$, tal que $e_{r e f}(k)=y(k)-y_{d}(k)$.

\section{Algoritmo FASS-NLMS}

Após desenvolvidas as equações de diferença que descrevem o comportamento dinâmico do IAIC, para que seja possível rastrear adaptativamente a dinâmica inversa da planta, é necessário, a cada instante de tempo, atualizar a estimava do vetor de pesos $\boldsymbol{\Theta}(k)$. O algoritmo FASS-NLMS atualiza a estimativa do vetor de pesos $\boldsymbol{\Theta}(k)$ através da seguinte formulação:

$$
\begin{aligned}
& \text { FASS-NLMS : } \mu(k)=\operatorname{MFIS}\left(e^{2}(k), \mathcal{K}(k)\right) \\
& \boldsymbol{\Theta}(k+1)=\left\{\begin{array}{l}
\boldsymbol{\Theta}(k)+\mu(k) \frac{e(k) \boldsymbol{\Psi}(k)}{\boldsymbol{\Psi}^{T}(k) \boldsymbol{\Psi}(k)}, \text { se } \boldsymbol{\Psi}^{T}(k) \boldsymbol{\Psi}(k) \neq 0, \\
\boldsymbol{\Theta}(k) \quad \text { se } \boldsymbol{\Psi}^{T}(k) \boldsymbol{\Psi}(k)=0
\end{array} \quad k \in[1, K]\right.
\end{aligned}
$$

As entradas do MFIS são o erro quadrático $e^{2}(k)$ e $\mathcal{K}(k)$ que é o instante de tempo normalizado pelo método Min-Max. Através da fuzzificação, as variáveis linguísticas de entrada do MFIS recebem um grau de pertinência pertencente ao intervalo $[0,1]$, por meio dos seguinte mapeamentos $m_{j}(\mathcal{K}(k)): \mathbb{R} \rightarrow[0,1]$ e $m_{j}\left(e^{2}(k)\right): \mathbb{R} \rightarrow[0,1]$. O mapeamento é realizado através da $j$-ésima função de pertinência (do inglês, MBF - Membership Function), de cada variável linguística, definida no universo de discurso. Para cada variável linguística de entrada do MFIS, foram definidas três MBFs do tipo triangular, com os valores linguísticos Pequeno "P"para $j=1$, Médio "M"para $j=2$ e Grande "G"para $j=3$. Na Tabela 1, são mostrados os parâmetros das MBFs que são definidas em função do conhecimento do especialista sobre como deve ser realizado o trade-off. Por meio de conectivos lógicos as proposições fuzzy que associam as variáveis linguísticas aos valores linguísticos são conectadas formando regras fuzzy do tipo Se antecedente então consequente. $\mathrm{Na}$ base de regras fuzzy desenvolvida para o FASS-NLMS, conforme pode ser visto em (6), as proposições fuzzy do antecedente são conectadas por conectivos lógicos "e" que realiza a seguinte operação:

$$
t\left[m_{j}(\mathcal{K}(k)), m_{j}\left(e^{2}(k)\right)\right]=\min \left[m_{j}(\mathcal{K}(k)), m_{j}\left(e^{2}(k)\right)\right],
$$

em que $t[\bullet]$ representada a norma-t. Através do cálculo da norma-t, que seleciona o valo mínimo entre $m_{j}(\mathcal{K}(k))$ e $m_{j}\left(e^{2}(k)\right)$, é obtido o grau de pertinência de ativação da $i$-ésima regra fuzzy.

$$
\begin{aligned}
& \mathcal{R}^{1}: \text { Se } \mathcal{K} \text { é } \mathrm{P} \text { e } e^{2} \text { é } \mathrm{P} \text { então } \omega^{1} \text { é } \mathrm{M} \\
& \mathcal{R}^{3}: \text { Se } \mathcal{K} \text { é } \mathrm{P} \text { e } e^{2} \text { é } \mathrm{G} \text { então } \omega^{3} \text { é } \mathrm{M} \\
& \mathcal{R}^{5} \text { : Se } \mathcal{K} \text { é } \mathrm{M} \text { e } e^{2} \text { é } \mathrm{M} \text { então } \omega^{5} \text { é } \mathrm{P} \\
& \mathcal{R}^{7} \text { : Se } \mathcal{K} \text { é } \mathrm{G} \text { e } e^{2} \text { é } \mathrm{P} \text { então } \omega^{7} \text { é } \mathrm{P} \\
& \mathcal{R}^{9}: \text { Se } \mathcal{K} \text { é } \mathrm{G} \text { e } e^{2} \text { é } \mathrm{G} \text { então } \omega^{9} \text { é } \mathrm{G}
\end{aligned}
$$

$$
\begin{aligned}
& \mathcal{R}^{2}: \text { Se } \mathcal{K} \text { é } \mathrm{P} \text { e } e^{2} \text { é } \mathrm{M} \text { então } \omega^{2} \text { é } \mathrm{M} \\
& \mathcal{R}^{4}: \text { Se } \mathcal{K} \text { é } \mathrm{M} \text { e } e^{2} \text { é } \mathrm{P} \text { então } \omega^{4} \text { é } \mathrm{P} \\
& \mathcal{R}^{6}: \text { Se } \mathcal{K} \text { é } \mathrm{M} \text { e } e^{2} \text { é } \mathrm{G} \text { então } \omega^{6} e^{\prime} \mathrm{G} \\
& \mathcal{R}^{8}: \text { Se } \mathcal{K} \text { é } \mathrm{G} \text { e } e^{2} \text { é } \mathrm{M} \text { então } \omega^{8} \text { é } \mathrm{M}
\end{aligned}
$$


Com a obtenção do grau de pertinência de ativação da $i$-ésima regra fuzzy logo em seguida é calculada a implicação fuzzy do consequente da $i$-ésima regra. A entrada da implicação fuzzy é o grau de pertinência de ativação da $i$-ésima regra fuzzy obtido no antecedente e a saída é uma MBF. Para obter a MBF de saída do consequente da $i$-ésima regra, é utilizado o operador mínimo. Uma vez que cada regra fuzzy é ativada com um determinado grau de pertinência, para se obter uma única $\mathrm{MBF}$ de saída as MBFs de todas as regras fuzzy precisam ser combinadas através da agregação fuzzy, para que o MFIS possa obter uma resposta total que é a MBF de saída. Após realizada a agregação, é necessário obter um valor numérico de saída que não seja um valor linguístico. Para isso, é realizada a defuzzificação do MBF de saída obtido pela agregação fuzzy. No algoritmo de otimização proposto, é utilizado o método de defuzzificação do tipo centroide, dado por:

$$
\mu(k)=\frac{\sum_{i=1}^{9} \mu^{i}(k) m_{j}\left(\mu^{i}(k)\right)}{\sum_{i=1}^{9} m_{j}\left(\mu^{i}(k)\right)},
$$

tal que para a variável linguística de saída do MFIS, que é $\mu(k)^{i}$, foram definidas três MBFs do tipo triangular, com os valores linguísticos Pequeno "P"para $j=1$, Médio "M"para $j=2$ e Grande "G" para $j=3$, de tal forma que os parâmetros das MBFs podem ser vistos na Tabela 1.

Tabela 1: Intervalo das MBFs triangulares.

\begin{tabular}{|c|c|c|c|c|c|}
\hline \multicolumn{2}{|l|}{$\mathcal{K}(k)$} & \multicolumn{2}{|c|}{$e^{2}(k)$} & \multicolumn{2}{|c|}{$\mu^{i}(k)$} \\
\hline Valor Linguístico & Intervalo & Valor Linguístico & Intervalo & Valor Linguístico & Intervalo \\
\hline Pequeno & {$\left[\begin{array}{ll}0 & 0,3\end{array}\right]$} & Pequeno & {$\left[0,1.10^{-5} \quad 0,9.10^{-5}\right]$} & Pequeno & {$\left[\begin{array}{lll}0,001 & 0,015]\end{array}\right]$} \\
\hline Médio & {$\left[\begin{array}{ll}0,2 & 0,5\end{array}\right]$} & Médio & {$\left[0,3 \cdot 10^{-5} 1 \cdot 10^{-5}\right]$} & Médio & {$\left[\begin{array}{lll}0,005 & 0,03]\end{array}\right.$} \\
\hline Grande & {$\left[\begin{array}{ll}0,3 & 1,0\end{array}\right]$} & Grande & {$\left[0,9 \cdot 10^{-5} 3.10^{-5}\right]$} & Grande & {$\left[\begin{array}{lll}0,015 & 0.1\end{array}\right]$} \\
\hline
\end{tabular}

\section{Resultados Computacionais}

Nessa seção, são apresentados os resultados computacionais obtidos através da aplicação do IAIC a uma planta estável e de fase não mínima. O sinal de distúrbio $n(k)$ foi adicionado ao sinal de controle $u(k)$. O modelo da planta $P\left(q^{-1}\right)$, o sinal de referência $r(k)$ e o sinal de distúrbio $n(k)$ foram obtidos em [7]. A equação de diferença da planta é dada por:

$$
y(k)=q^{-1} \frac{1-3 q^{-1}+3,5 q^{-2}}{1+0,05 q^{-2}+0,05 q^{-3}+0,02 q^{-4}}(u(k)+n(k)),
$$

no qual a planta é estável com zeros ${ }^{4}$ localizados em $1,500 \pm \bar{j} 1,1180$ e polos ${ }^{5}$ em $0,2500 \pm \bar{j} 0,3708$ $\mathrm{e}-0,2500 \pm \bar{j} 0,1936$. O período de amostragem foi definido como $0,001 \mathrm{~s}{ }^{6}$. O tempo de simulação foi de $30 \mathrm{~s}$, sendo assim o número total de instantes de tempo ou amostras foi de $K=30000$. Com o objetivo de comparar os desempenhos dos algoritmos FASS-NLMS e NLMS no projeto do IAIC, o tamanho de passo para o algoritmo NLMS foi definido com $\mu=0,01$, conforme proposto em [7].

A ordem do filtro FIR adaptativo $\hat{C}\left(q^{-1}\right)=\hat{C}_{\text {cópia }}\left(q^{-1}\right)$ foi definida com $M=10$. O bloco de atraso $q^{-L}$ foi definido com $L=7$. O sinal de referência $(k)$ foi definido com uma periodicidade de $5 s$ entre os degraus de referência de 1 e -1 . A comparação entre o rastreamento do sinal de referência realizado pelo IAIC projetado pelos algoritmos FASS-NLMS e NLMS é mostrado na Figura 2. É possível notar que, mesmo na presença do sinal de distúrbio $n(k)$, o rastreamento da dinâmica inversa da planta realizada pela atualização da estimativa do vetor de pesos $\boldsymbol{\Theta}(k)$ do filtro

\footnotetext{
${ }^{4}$ Os zeros são as raízes do polinômio do numerador.

${ }^{5}$ Os polos são as raízes do polinômio do denominador.

${ }^{6}$ Período de amostragem é o período de tempo entre cada amostra obtida de um sinal.
} 


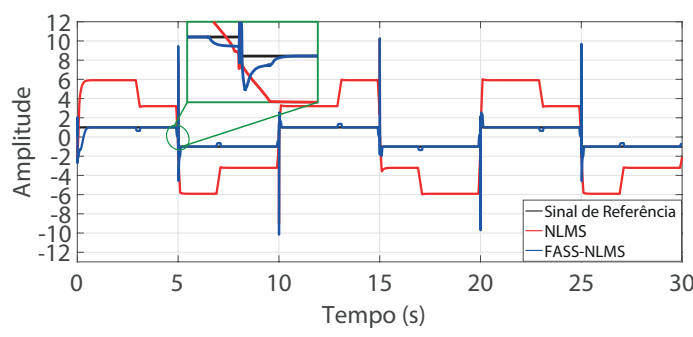

Figura 2: Sinal de saída da planta $y(k)$.

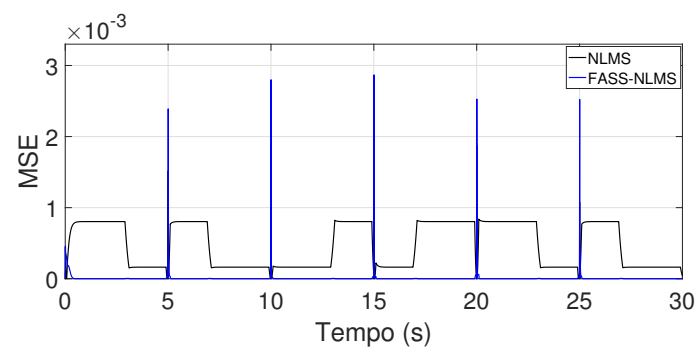

Figura 4: MSE de $e_{r e f}(k)$.

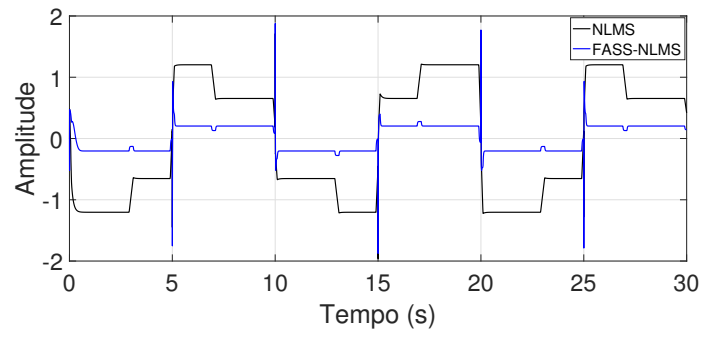

Figura 3: Sinal de controle $u(k)$.

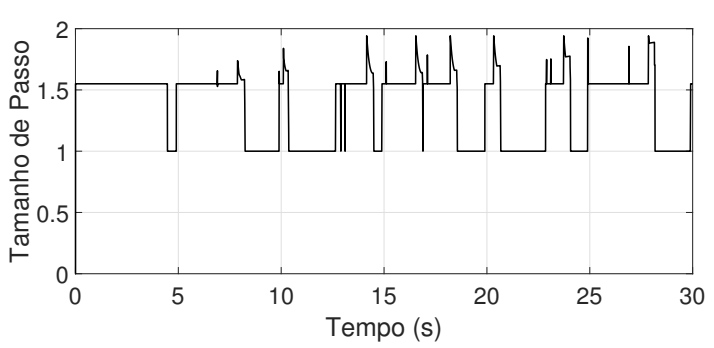

Figura 5: Atualização do tamanho de passo.

FIR adaptativo $\hat{C}\left(q^{-1}\right)$, permitiu que o IAIC projetado pelo algoritmo FASS-NLMS desenvolvesse uma boa performance. Embora o sinal de saída da planta $y(k)$, para o IAIC projetado pelo algoritmo FASS-NLMS, tenha apresentado um overshoot na mudança do degrau de referência, nota-se que convergiu rapidamente para o sinal de referência $r(k)$ e com um MSE no regime estacionário igual a zero, conforme mostrado nas Figuras 2 e 4 . Esse comportamento não foi desenvolvido pelo IAIC projetado pelo algoritmo NLMS, no qual foi observado que o sinal de saída da planta $y(k)$ não convergiu para o sinal de referência $r(k)$.

É possível notar, conforme mostrado na Figura 3, que o IAIC projetado pelo algoritmo FASSNLMS desenvolveu um sinal de controle $u(k)$ com uma rápida e forte ação de controle, que é resultado do satisfatório rastreamento da dinâmica inversa da planta pelo filtro FIR adaptativo $\hat{C}\left(q^{-1}\right)$. Esse resultado favoreceu a uma rápida convergência do sinal de saída da planta $y(k)$ para o sinal de referência $r(k)$. Na Figura 5 , é mostrado o tamanho de passo adaptado pelo MFIS para o algoritmo FASS-NLMS. De acordo com a base regras fuzzy, o tamanho de passo é adaptado em função do erro quadrático e do instante de tempo normalizado, sendo possível notar que os valores obtidos na adaptação do tamanho de passo permaneceram no intervalo de estabilidade $0<\mu(k)<2$ do algoritmo NLMS. Dessa forma, a cada instante de tempo, a descida do gradiente estocástico, através da adaptação do tamanho de passo, foi realizada com objetivo de obter um bom trade-off entre a velocidade de convergência e o MSE no regime estacionário. O bom trade-off pode ser verificado através da rápida convergência do sinal de saída da planta $y(k)$ para o sinal de referência $r(k)$ e do MSE no regime estacionário igual a zero, mostrados nas Figuras 2 e 4.

\section{Conclusão}

O algoritmo FASS-NLMS proposto nesse trabalho, foi avaliado no projeto de IAIC em uma planta de fase não mínima na presença de um sinal de distúrbio. Nos resultados obtidos foi observado que através da adaptação do tamanho de passo por um MFIS, um bom trade-off entre 
a velocidade de convergência e o MSE no regime estacionário foi obtido, confirmado através da satisfatória e rápida convergência do sinal de saída da planta para o sinal de referência. Além disso, foi observado que devido a adaptação do tamanho de passo através do MFIS, mesmo após a mudança de degrau do sinal de distúrbio, o sinal de saída da planta ainda continuou a convergir para o sinal de referência, mostrando uma capacidade de rejeição de distúrbio. Ainda, é possível notar que a adaptação do tamanho de passo pelo MFIS é independente de medidas estatísticas de alta ordem e da natureza do problema. Sendo assim, o algoritmo FASS-NLMS é apto a trabalhar em um contexto de aplicação em tempo real.

\section{Referências}

[1] Bai, L., Yin, Q. A modified NLMS algorithm for adaptive noise cancellation, 2010 IEEE International Conference on Acoustics, Speech and Signal Processing, 3726-3729, 2010. DOI:10.1109/ICASSP.2010.5495868.

[2] Benesty, J., Rey, H., Vega, L. R., Tressens, S. A Nonparametric VSS NLMS Algorithm, IEEE Signal Processing Letters, 13:581-584, 2006. DOI: 10.1109/LSP.2006.876323.

[3] Ferrer, M., Diego, M., Piñero, G., Gonzalez, A. Affine Projection Algorithm Over Acoustic Sensor Networks for Active Noise Control, IEEE/ACM Transactions on Audio, Speech, and Language Processing, 29:448-461, 2020. DOI: 10.1109/TASLP.2020.3042590.

[4] Ng, Y. H., Mohamad, H., Chuah, T. C. Block-based fuzzy step size NLMS algorithms for subband adaptive channel equalisation, IET Signal Processing, 3:23-32, 2009. DOI: 10.1049/ietspr:20070222.

[5] Kim, D. W., Hur, J., Park, P. Two-stage active noise control with online secondary-path filter based on an adapted scheduled-stepsize NLMS algorithm, Applied Acoustics, 158:107031, 2020. DOI: $10.1016 /$ j.apacoust.2019.107031.

[6] Pauline, S. H., Samiappan, D., Kumar, R., Anand, A., Kar, A. Variable tap-length nonparametric variable step-size NLMS adaptive filtering algorithm for acoustic echo cancellation, Applied Acoustics, 159:107074, 2020. DOI: 10.1016/j.apacoust.2019.107074.

[7] Shafiq, M., Shafiq, M. A., Yousef, H. A. Stability and Convergence Analysis of Direct Adaptive Inverse Control, Complexity, 2017, DOI: 10.1155/2017/7834358.

[8] Tavoosi, J. An experimental study on inverse adaptive neural fuzzy control for nonlinear systems, International Journal of Knowledge-based and Intelligent Engineering Systems, 24:135143, 2020. DOI: 10.3233/KES-200036.

[9] Orozco-Tupacyupanqui, W., Nakano-Miyatake, M., Perez-Meana, H. A Novel Neural-Fuzzy Method to Search the Optimal Step Size for NLMS Beamforming, IEEE Latin America Transactions, 13:402-408, 2015. DOI: 10.1109/TLA.2015.7055556.

[10] Wang, H., Pi, D., Sun, Y. Online SVM regression algorithm-based adaptive inverse control, Neurocomputing, 70:952-959, 2007. DOI: 10.1016/j.neucom.2006.10.021.

[11] Wang, X. Y., Wang, Y., Li, Z. S. Research of the 3-DOF Helicopter System Based on Adaptive Inverse Control, Applied Mechanics and Materials, 389:623-631, 2013. DOI: 10.4028/www.scientific.net/AMM.389.623.

[12] Widrow, B., Walach, E. Adaptive signal processing for adaptive control, IFAC Proceedings Volumes, 16:7-12, 1983. DOI: 10.1016/S1474-6670(17)62348-6. 